\title{
Analysis of near-IR spectra of 1 Ceres and 4 Vesta, targets of the Dawn mission
}

\author{
P. Vernazza ${ }^{1}$, T. Mothé-Diniz ${ }^{1}$, M. A. Barucci ${ }^{1}$, M. Birlan ${ }^{2}$, J. M. Carvano ${ }^{1}$, \\ G. Strazzulla ${ }^{3}$, M. Fulchignoni ${ }^{1}$, and A. Migliorini ${ }^{1,4}$ \\ 1 LESIA, Observatoire de Paris, 92195 Meudon Principal Cedex, France \\ e-mail: pierre.vernazza@obspm.fr \\ 2 IMCCE, Observatoire de Paris, 77 Av. Denfert Rochereau, 75014 Paris Cedex, France \\ 3 INAF-Osservatorio Astrofisico di Catania, via Santa Sofia 78, 95123 Catania, Italy \\ ${ }^{4}$ Dipartimento di Astronomia di Padova, Vicolo dell'Osservatorio 2, 35122 Padova, Italy
}

Received 8 December 2004 / Accepted 3 February 2005

\begin{abstract}
We obtained high signal to noise spectra of the two targets of the Dawn mission, 4 Vesta and 1 Ceres from observations carried out in remote control between the Observatoire de Paris-Meudon and the NASA Infrared Telescope Facility on Mauna Kea. 4 Vesta was observed in the $0.7-2.5 \mu \mathrm{m}$ spectral region at three different rotational phases in order to i) determine the mineral composition; ii) understand the spectral variations across the surface. Vesta was also observed in the 2.0-3.8 $\mu \mathrm{m}$ range. The $3 \mu \mathrm{m}$ absorption feature was not detected, implying the absence of $\mathrm{OH}$ and/or $\mathrm{H} 2 \mathrm{O}$-bearing minerals on the asteroid surface at the latitude of our observations. The spectrum of 1 Ceres was obtained in the $2.0-4.1 \mu \mathrm{m}$ range and the presence of the $3.06 \mu \mathrm{m}$ absorption feature confirmed. Laboratory measurement of ion-irradiated organics and ices suggest that the $3.06 \mu \mathrm{m}$ feature can be reproduced with a linear mixture of crystalyne ice and residues of ion-irradiated asphaltite.
\end{abstract}

Key words. minor planets, asteroids - techniques: spectroscopic - infrared: solar system

\section{Introduction}

4 Vesta and 1 Ceres are the two asteroids which will be visited by the Dawn space mission. Dawn is a NASA Discovery mission scheduled to be launched in June 2006 arriving at Vesta in 2011 and at Ceres in 2015. The spacecraft will orbit for several months around each object with the aim of understanding the origin and evolution of the solar system by obtaining geophysical and geochemical data on these diverse main belt asteroids. Ceres is the largest asteroid with a mean diameter of $950 \mathrm{~km}$, while Vesta is the third largest asteroid with a mean diameter of $516 \mathrm{~km}$. They are among the most massive asteroids: 4 Vesta $\left(2.75 \times 10^{20} \mathrm{~kg}\right)$ and 1 Ceres $\left(9.43 \times 10^{20} \mathrm{~kg}\right)$ (Standish \& Hellings 1989). They could be considered as small planets and due to their location, they could have experienced many of the processes that are generally associated with the planetary evolution. Ceres is an undifferentiated protoplanet while Vesta has experienced significant heating and differentiation. Vesta and Ceres were formed by accretion of smaller objects over short time scales, and even if they are supposed to have been formed at relatively close areas of the main belt, they are very different objects.

Vesta is the only large asteroid known to have a basaltic surface that retains a record of ancient volcanic activity. The basaltic nature on Vesta was discovered through visible spectroscopy with ground-based telescopes (McCord et al. 1970).
The Vesta unique reflectance spectrum and its high albedo 0.42 (Tedesco 1989) compared to other asteroids defined a new taxonomic class (type V). Geological diversity revealing longitudinal variations in albedo and mineralogy was detected from various observational techniques obtained at different rotational phases and aspect angles. The high geological interest on this asteroid followed the discovery that howardite, eucrite, and diogenite (HED) meteorites could be samples excavated from Vesta. In 1996, Vesta made its closest approach to Earth and observations with WFPC2 (HST) allowed images to be obtained at a scale of $36 \mathrm{~km} /$ pixel thus providing coverage of the southern hemisphere (Thomas et al. 1997). The surface of Vesta is not uniform, showing some dark and bright areas. The Hubble Space Telescope has also revealed a very large, circular cavity near the asteroid's south pole, and the 460-km-wide basin has a pronounced central peak of $12 \mathrm{~km}$. Its diverse surface makes Vesta one of the most geologically peculiar large asteroids; it is so exceptional that its origin and history are far from being understood till now. See Keil (2002) for a review of our present knowledge of Vesta.

We know even less about the composition of Ceres, which has a low albedo 0.11 (Tedesco 1989), a quasi-spherical shape, and few spectral features. The reflectance spectrum is flat and featureless in the visible with an absorption band near $3 \mu \mathrm{m}$ associated to hydrated minerals (Lebofsky et al. 1981) and/or ammoniated saponite by King et al. (1992). Microwave 
Table 1. Observational circumstances for 4 Vesta and 1 Ceres with the associated phase angle $\left(\alpha_{\mathrm{s}}\right)$, longitude $(W)$, latitude $(\zeta)$, and rotational phase $\left(\Phi_{W}\right)$.

\begin{tabular}{|c|c|c|c|c|c|c|c|}
\hline \multicolumn{8}{|c|}{4 VESTA } \\
\hline Day & UT & $\lambda(\mu \mathrm{m})$ & a.m. & $\alpha_{\mathrm{s}}$ & $W$ & $\zeta$ & $\Phi_{W}$ \\
\hline 30 Mar. 03 & 10:03-10:04 & $0.7-2.5$ & 1.02 & 5.8 & $238^{\circ}$ & $-11^{\circ}$ & 0.06 \\
\hline 30 Mar. 03 & $12: 16-12: 17$ & $0.7-2.5$ & 1.13 & 5.8 & $27^{\circ}$ & $-11^{\circ}$ & 0.475 \\
\hline 30 Mar. 03 & $13: 10-13: 26$ & $0.7-2.5$ & 1.34 & 5.8 & $88^{\circ}$ & $-11^{\circ}$ & 0.64 \\
\hline 31 Mar. 03 & $10: 05-10: 35$ & $2.0-4.1$ & 1.02 & 6.0 & $57^{\circ}-91^{\circ}$ & $101^{\circ}$ & $0.56-0.65$ \\
\hline 31 Mar. 03 & $11: 29-11: 51$ & $2.0-4.1$ & 1.05 & 6.0 & $152^{\circ}-176^{\circ}$ & $101^{\circ}$ & $0.82-0.89$ \\
\hline \multicolumn{8}{|c|}{1 CERES } \\
\hline 25 Jan. 04 & $6: 41-6: 58$ & $2.0-4.1$ & 1.2 & & & & \\
\hline 25 Jan. 04 & $7: 18-7: 35$ & $2.0-4.1$ & 1.2 & & & & \\
\hline
\end{tabular}

studies suggest that Ceres is covered by a dry clay that reflects its crustal composition (Webster et al. 1988). There are no clear links between Ceres and any known meteorite, and McCord \& Sotin (2003) suggested that the thermal evolution of Ceres could have produced a metamorphosed carbonaceous chondrite surface material, thereby also producing a surface with an albedo higher than the original materials. Recent publications (Parker et al. 2002; Britt et al. 2002) summarize the present knowledge of Ceres. Ceres is a mysterious and probably unique object in the solar system representative of the early phase of planet formation.

In this work we present new spectra of Vesta in the nearinfrared $(0.7-2.5 \mu \mathrm{m}$ and $2.0-4.1 \mu \mathrm{m})$ obtained at various rotation phases and a spectrum in the range $2.0-4.1 \mu \mathrm{m}$ for Ceres. These results are analysed and discussed.

\section{Observations and data reduction}

\subsection{Observations}

The spectra presented in this work were obtained at the Infrared Telescope Facility (IRTF), a $3 \mathrm{~m}$ telescope located at the summit of Mauna Kea, Hawaii. Observations were remotely conducted from Meudon, France, more than $12000 \mathrm{~km}$ away from Hawaii, using several informational structures and networks. This type of observation between Meudon and IRTF/Hawaii was started in 2002 (Birlan et al. 2002); since then, more than thirty nights of observation were conducted from Meudon.

Two observing runs in March 2003 and January 2004 were devoted to near-infrared observation of 4 Vesta and 1 Ceres, respectively. The spectrograph SpeX, combined with the $0.8 \times$ 15 arcsec slit, were used for acquisition of spectra in the range $0.7-4.1 \mu \mathrm{m}$. All observations were made with pairs of exposures named "A" and "B", which allowed us to produce simultaneous measurements of the object and the sky.

4 Vesta was observed on March 30 in LowRes $(R=$ 200) prism mode for the spectral range $0.7-2.5 \mu \mathrm{m}$, while spectra in the range $2.0-4.1 \mu \mathrm{m}$ (LXD mode, $R=2000$ ) were acquired on March 31. In order to monitor the asteroid during its rotational phase, several series of spectra were obtained. The observational circumstances for Vesta are presented in
Table 1, which lists the date and time for each observation and the wavelength range, $V$ magnitude, airmass, and solar phase angle $\left(\alpha_{\mathrm{s}}\right)$. This table also shows the sub-earth longitude of Vesta $(W)$, the sub-earth latitude $(\zeta)$, calculated using the pole solution of Thomas et al. (1997), and the rotational phase calculated with respect to the zero phase of Gaffey (1997) - hereafter G97 - extrapolated using Thomas et al. (1997) and Binzel et al. (1997). Unless otherwise stated, the Vesta spectra will be referred to mainly by the rotational phase. During both nights the atmospheric conditions were not stable with the occasional presence of thin clouds. This problem was carefully taken into account in data-reduction (see Sect. 2.2). During these nights, the humidity range was $25 \%-55 \%$, and the values of the seeing were in the range $0.8-1.5 \mathrm{arcsec}$, while the temperature variations were between $-1{ }^{\circ} \mathrm{C}$ and $+3.2^{\circ} \mathrm{C}$. The observations of 4 Vesta were intercalated with those of standard stars Landolt 102-1081, HD 88618, Landolt 107-684, Landolt 105-56, Landolt 107-998, Hya 64, and Landolt 98-978.

The asteroid 1 Ceres was observed on January 25, 2004 in the spectral range 2.0-4.1 micron (LXD mode, $R=2000$ ). Observational circumstances are presented in Table 1. For calibration of our spectra, these observations were alternated with those of Hya 64 and SAO 95394 standard stars. During these observations the humidity rate was high, almost $90 \%$, the temperature stable at $-4{ }^{\circ} \mathrm{C}$, and the seeing measurement was 1.1 arcsec.

\subsection{Data reduction}

Standard techniques for near-IR spectroscopy reduction were used with the software Spextool 3.2 dedicated to reducing data obtained with Spex. We started by creating normalized flatfields for each observing night, then subtracted image pairs taken at two different positions (A and B) along the slit, in order to minimize the atmospheric and telescope influence and to remove the influence of electronic bias level and the dark current. Each background subtracted image was divided by the flat field image to remove pixel to pixel sensitivity variation on the chip. Then the data were extracted from two-dimensional images to one-dimensional arrays by summing pixels in the data 
range for each column. Finally, we performed the wavelength calibration of the spectra. Telluric absorptions were removed by dividing the asteroid spectra by stellar spectrum at the same airmass. We used a star observed close in airmass $( \pm 0.03)$ to make the division for both spectra at $10 \mathrm{~h} 03 \mathrm{UT}$ and $12 \mathrm{~h} 16 \mathrm{UT}$ (Table 1).

For the third spectrum of Vesta, observed at an airmass of 1.34 , we had no star close in airmass to make a good correction concerning the extinction coefficients. To perform the correction for extinction, we used observations of a star obtained at a different period (IRTF, November 2003) but at the same airmass. To ensure that this process did not introduce any significant error, we divided the standard stars observed during the observation of Vesta by those stars with corresponding airmass observed on November 2003 and spanning airmasses from 1.03 to 1.2. This division always produced a flat spectrum, which shows that the extinction coefficients did not vary considerably between the two nights. Nevertheless, the depth of the water vapour absorption bands were different from one night to the next due to a difference in degree of humidity. To correct for humidity, we used a so-called transmission spectrum (spectrum of a star observed the same night as Vesta divided by a star observed in November with same airmass for both stars) in the water zones. Thus, to obtain the final reflectance we divided the spectra of Vesta by a star obtained during November at same airmass to correct for extinction and multiplied by the "transmission" spectra in the water zones. The reduced spectra of Vesta in this range are shown in Fig. 1

We used the LXD mode $(2.0-4.1 \mu \mathrm{m})$ fitting onto the array at a spectral resolving power of $R=2000$, with a slit length of $15^{\prime \prime}$, and a slit width of $0.8^{\prime \prime}$. The $2.0-4.1 \mu \mathrm{m}$ region is covered by two pieces, one from $2.0-2.55 \mu \mathrm{m}$ and another from $2.85-4.1 \mu \mathrm{m}$. The LXD mode allows simultaneous observations on both sides of the telluric water band at $2.55-2.85 \mu \mathrm{m}$. The $2.0-4.1 \mu \mathrm{m}$ region is covered by 6 overlapping spectra. While relative fluxes within one observation will be correct, absolute fluxes cannot be easily compared from one integration to the next and must be scaled to one another.

In order to have the reflectance spectrum of Vesta and Ceres, we corrected for thermal flux. Thermal radiation in the main belt starts to affect data in the $3-\mu \mathrm{m}$ region. To remove this thermal contribution, we used the standard thermal model developed by Lebofsky \& Spencer (1989). This model assumes the situation of a non-rotating spherical asteroid in instantaneous equilibrium with solar flux.

For Vesta, thermal contribution to the total reflectance was less than $0.01 \%$ at $3.0 \mu \mathrm{m}$ and $8 \%$ at $3.8 \mu \mathrm{m}$, and for Ceres less then $0.3 \%$ at $3.0 \mu \mathrm{m}$ and $70 \%$ at $4.1 \mu \mathrm{m}$. Of course, the thermal corrections remain model-dependent and will noticeably affect the interpretation of our corrected spectra.

\section{Results and discussion}

\subsection{Vesta}

The spectra of Vesta in the $0.7-2.5 \mu \mathrm{m}$ and in the $2.0-3.8 \mu \mathrm{m}$ range are discussed separately, since each range contains specific mineralogical information and requires quite different

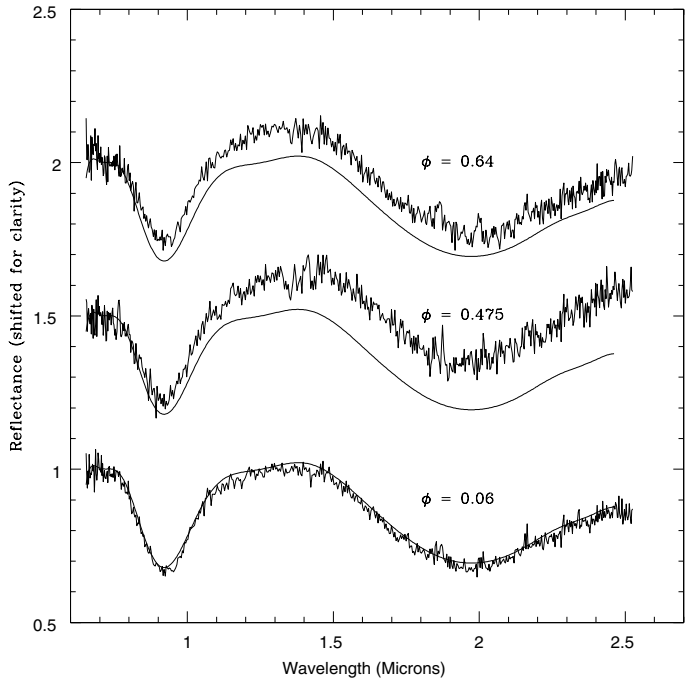

Fig. 1. Spectra of Vesta at each rotational phase, compared with the fit of the spectrum at $\Phi=0.06$ (continuous line). The spectra were vertically displaced for clarity by 0.5 .

analytical approaches. The main silicate features reside in the $0.7-2.5 \mu \mathrm{m}$ range that are part of the diagnosis of basaltic compositions. Such spectra are analysed here using both empirical laboratory calibrations (Cloutis et al. 1986; Cloutis \& Gaffey 1991) and the Modified Gaussian Model (Sunshine et al. 1990). The $2.0-3.8 \mu \mathrm{m}$ range, on the other hand, is potentially interesting since the detection of features around $3 \mu \mathrm{m}$ and at $3.4 \mu \mathrm{m}$ could reveal the presence of $\mathrm{OH} / \mathrm{H}_{2} \mathrm{O}$ and $\mathrm{CH}$-bearing minerals.

\subsubsection{Silicate mineralogy $(0.7-2.5 \mu \mathrm{m})$}

A widely used methodology for mineralogical interpretation of reflectance spectra in olivine/pyroxene assemblages is the use of empirical formulae derived from laboratory calibrations. This method has recently been reviewed by Gaffey et al. (2002). In order to apply it, the spectra must be parameterised in terms of the areas and position of the absorption band centers located near 1 and $2 \mu \mathrm{m}$, which from now on will be referred as $\mathrm{BI}$ and BII, respectively. The centers and areas of BI and BII are measured after removal of a linear continuum that is tangent to the maxima before and after each band. These values are then inserted into empirical formulae from which the mineralogical composition of the pyroxenes in the assemblage in terms of percentage of Wolastonite (Wo) and Ferrosilite (Fs) content can be also derived along with the contribution of olivine in the mixture. It is important to note that these calibrations assume the presence of single pyroxene and would fail if more than one kind of pyroxene were present.

Using a polynomial fit of the full spectra between $0.7-2.5 \mu \mathrm{m}$, we determined the three maxima near $0.7,1.4$ and $2.5 \mu \mathrm{m}$. The linear continuum for $\mathrm{BI}$ is given by the straight line tangent to the maxima near 0.7 and $1.4 \mu \mathrm{m}$, while for BII the continuum is determined by the maxima around 1.4 and $2.5 \mu \mathrm{m}$. For each band, the continuum was removed by dividing each band by the linear continuum in each corresponding 
Table 2. 4 Vesta spectral parameters.

\begin{tabular}{cccccc}
\hline \hline $\begin{array}{c}\text { Observation } \\
\Phi\end{array}$ & $\begin{array}{c}\text { Band I } \\
(\mu \mathrm{m})\end{array}$ & $\begin{array}{c}\text { Band II } \\
(\mu \mathrm{m})\end{array}$ & BAR & $\begin{array}{c}\text { Wo } \\
(\mathrm{mol} \%)\end{array}$ & $\begin{array}{c}F \mathrm{~s} \\
(\mathrm{~mol} \%)\end{array}$ \\
\hline 0.06 & 0.926 & 1.958 & 2.05 & $6-7$ & 41 \\
0.47 & 0.922 & 1.932 & 2.35 & $5-6$ & 34 \\
0.64 & 0.926 & 1.996 & 2.68 & $6-7$ & 51 \\
\hline
\end{tabular}

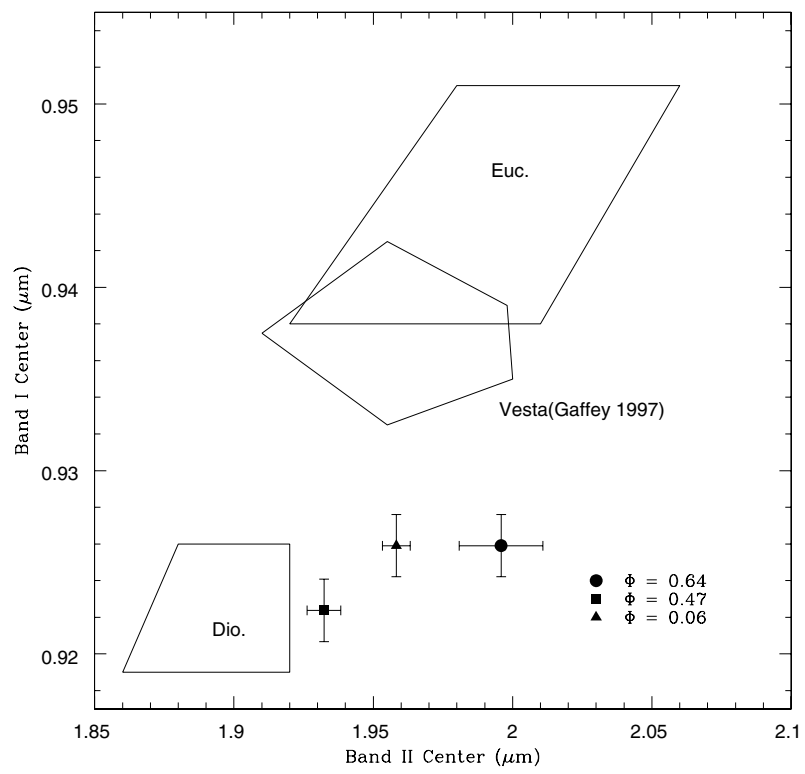

Fig. 2. Position of the band center for the three spectra of Vesta compared to the regions encompassed by the Eucrite (Euc.) and Diogenite (Dio.) basaltic achondrite assemblages and to the region encompassed by the observations of Vesta from G97. Different symbols represent different rotational phases of Vesta.

region. The center of each band was computed after the continuum removal, using polynomial fits around the region of each reflectance minimum, with orders varying from 3 to 6 . The adopted values for band centers correspond to the polynomial that yields the best fit, and the uncertainty in band center position is given by the scatter due to the use of different polynomials. The areas of BI and BII are also measured after removing the continua, and the parameter BAR is defined as the ratio between the areas of BII and BI. Using these parameters, the pyroxene composition in terms of Wo and Fs was derived for each spectrum through the equations recently recompiled by Gaffey et al. (2002). The spectral parameters and pyroxene composition for each spectrum are presented in Table 2. Figure 2 shows the position of the center of BI versus the center of BII for the spectra of Vesta in the present work, along with the loci of the eucrite and diogenite meteorites (Duffard et al. 2004a) and the loci of G97 observations for Vesta.

For all spectra, the position of the centers of BI and BII fall in the transition region between low- (orthopyroxene), and high-calcium pyroxenes (clinopyroxene) close to the diogenite locus and could be consistent with a single pyroxene composition. The derived values for Wo and Fs indicate that orthopyroxene is the dominant mafic mineral. The BAR values, when used to derive the percentage of orthopyroxene in

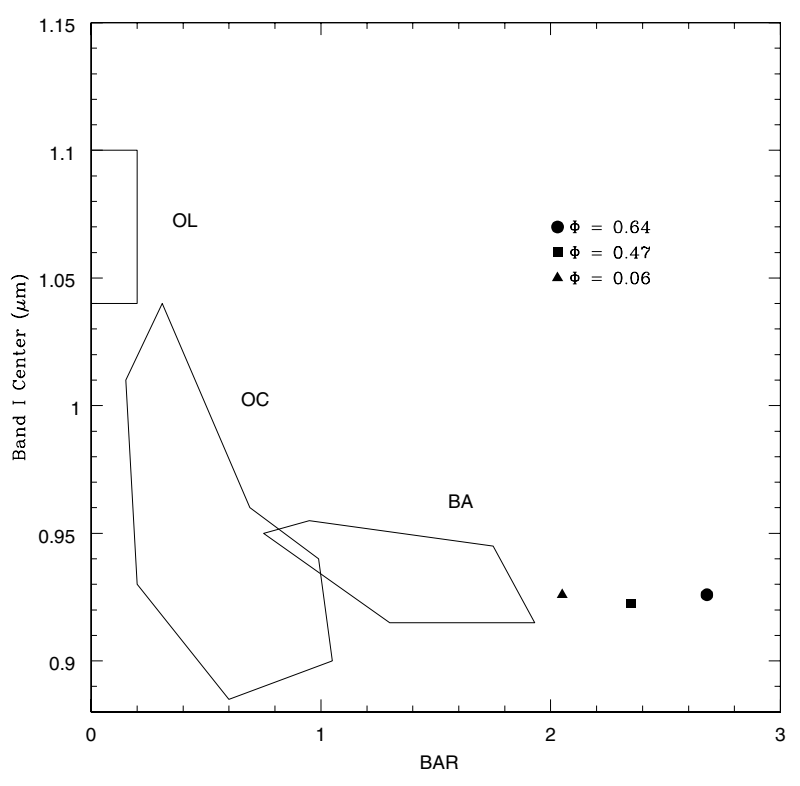

Fig. 3. The BAR value versus Band I center is represented for our observations at different rotational phases; error bars are smaller than the symbols. The loci correspond to: the monomineralic olivine assemblages (OL), the ordinary chondrites (OC), and the pyroxene basaltic achondrites (BA) (Duffard et al. 2004a).

orthopyroxene-olivine mixtures, along with the calibration of Cloutis et al. (1986), yield values in excess of $90 \%$ orthopyroxene, which are outside the validated range for this calibration. In fact, for rotational phases 0.47 and 0.64 this calibration produces values in excess of $100 \%$, which suggests that olivine is at most an accessory mineral phase, but it can also indicate a clinopyroxene phase.

On the BAR $\times$ BI center diagram (Fig. 3) our spectra are located on the edge of the basaltic achondrite loci, close to loci of pure orthopyroxene, which suggests that our spectra are dominated by low-calcium pyroxene. The spectral variations observed among rotational phases are mainly due to shifts in BII centers.

Although the position of those spectra in the band center diagram and in the $\mathrm{BI} \times \mathrm{BAR}$ diagram are consistent with the presence of a low calcium pyroxene, a composition consisting of a mixture of low calcium- and high-calcium pyroxenes cannot be ruled out. However, two pyroxenes, orthopyroxene and pigeonite, have their band centers falling in this region. Furthermore, our band centers are in the transition region between diogenites and eucrites. The primary mineral of diogenites is orthopyroxene (92-95\%), while the main pyroxene found in eucrites is pigeonite. Thus regarding the position of our band centers we should have both orthopyroxene and pigeonite with a much stronger contribution from orthopyroxene.

In order to check the effects of such a mixture on mineralogical interpretation of our data, we made use of the Modified Gaussian Model. The MGM model deconvolves spectra into a series of modified Gaussian curves, each representing an individual electronic transition absorption band. Mathematically, each absorption band is represented by three parameters: a band strength, a band center, and a band width. The absorption bands are all superimposed onto a continuum, which is modelled as 
Table 3. Parameters from MGM applied to 4 Vesta using the bands associated to the Orthopyroxenes in the range $0.6-2.6 \mu \mathrm{m}$ as described in Sunshine \& Pieters (1993).

\begin{tabular}{ccccc}
\hline \hline & $\begin{array}{c}\text { Center } \\
\Phi=0.06\end{array}$ & $\begin{array}{c}F W H M \\
(\mu \mathrm{m})\end{array}$ & $\begin{array}{c}\text { Strength } \\
(\text { natural log) }\end{array}$ & $\begin{array}{c}\text { Continuum and } \\
\text { Error in fit (rms) }\end{array}$ \\
\hline Band 1 & 0.678 & 0.149 & -0.11 & \\
Band 2 & 0.918 & 0.200 & -0.50 & Offset:1.1 \\
Band 3 & 1.161 & 0.280 & -0.14 & Slope:1.2E-007 \\
Band 4 & 1.962 & 0.660 & -0.49 & \\
Band 5 & 2.499 & 0.502 & -0.12 & rms: 2.53\% \\
\hline$\Phi=0.47$ & & & & \\
\hline Band 1 & 0.676 & 0.1477 & -0.2 & \\
Band 2 & 0.911 & 0.206 & -0.49 & Offset:1.19 \\
Band 3 & 1.148 & 0.278 & -0.11 & Slope:1.2E-007 \\
Band 4 & 1.946 & 0.582 & -0.35 & \\
Band 5 & 2.499 & 0.501 & -0.05 & rms: 3.3\% \\
\hline$\Phi=0.64$ & & & & \\
\hline Band 1 & 0.690 & 0.151 & -0.18 & \\
Band 2 & 0.915 & 0.211 & -0.49 & Offset:1.14 \\
Band 3 & 1.158 & 0.280 & -0.15 & Slope:1.2E-007 \\
Band 4 & 1.97 & 0.651 & -0.48 & \\
Band 5 & 2.499 & 0.504 & -0.10 & rms: 3.34\% \\
\hline
\end{tabular}

a straight line in energy and represented by two additional parameters, a slope and an offset (Sunshine et al. 1990).

A preliminary analysis was made using only low calcium pyroxene (LCP) bands. Five bands were used (Sunshine \& Pieters 1993), with band centers ranging from 0.6 to $2.5 \mu \mathrm{m}$. The band around $0.67 \mu \mathrm{m}$ was included in order to minimize the residua at lower wavelengths, even though most of this band lies outside our spectral range. Final values for the centers, widths, strengths, and rms are shown in Table 3. Residuals of the fits have low rms and show no systematic deviation from zero. Even so, a high calcium pyroxene (HCP) component cannot be excluded. We then applied the MGM by adding two clinopyroxene bands to the model. As shown in Fig. 4, the two pyroxene model also results in good fits for all three spectra of Vesta. On average, the additional pyroxene reduced the residual error, but the fits obtained are non-unique. The observed dispersion for band centers are on the order of $0.05 \mu \mathrm{m}$ and around $50 \%$ for some of the band strength. The parameter of representative fits are shown in Table 4 and resulting fits are also reported in Fig. 4. Using the calibration of Sunshine \& Pieters (1993), from the LCP/HCP band strengths, the rotational phase 0.47 has a $\mathrm{HCP} /(\mathrm{HCP}+\mathrm{LCP})$ ratio of $\sim 0.15-0.20$, while phases 0.06 and 0.64 have a ratio in the $\sim 0.17-0.38$ range, which is consistent with previous application of the MGM to an eucrite meteorite (Sunshine et al. 2004). However mineralogical analysis of HED meteorites show that they contain three kinds of pyroxenes: orthopyroxene, pigeonite, and augite. Since orthopyroxene and pigeonite have rather close band centers, it is not possible to meaningfully deconvolve them with MGM. Therefore the LCP phase found by MGM could suggest the presence of one of those phases or a mixture
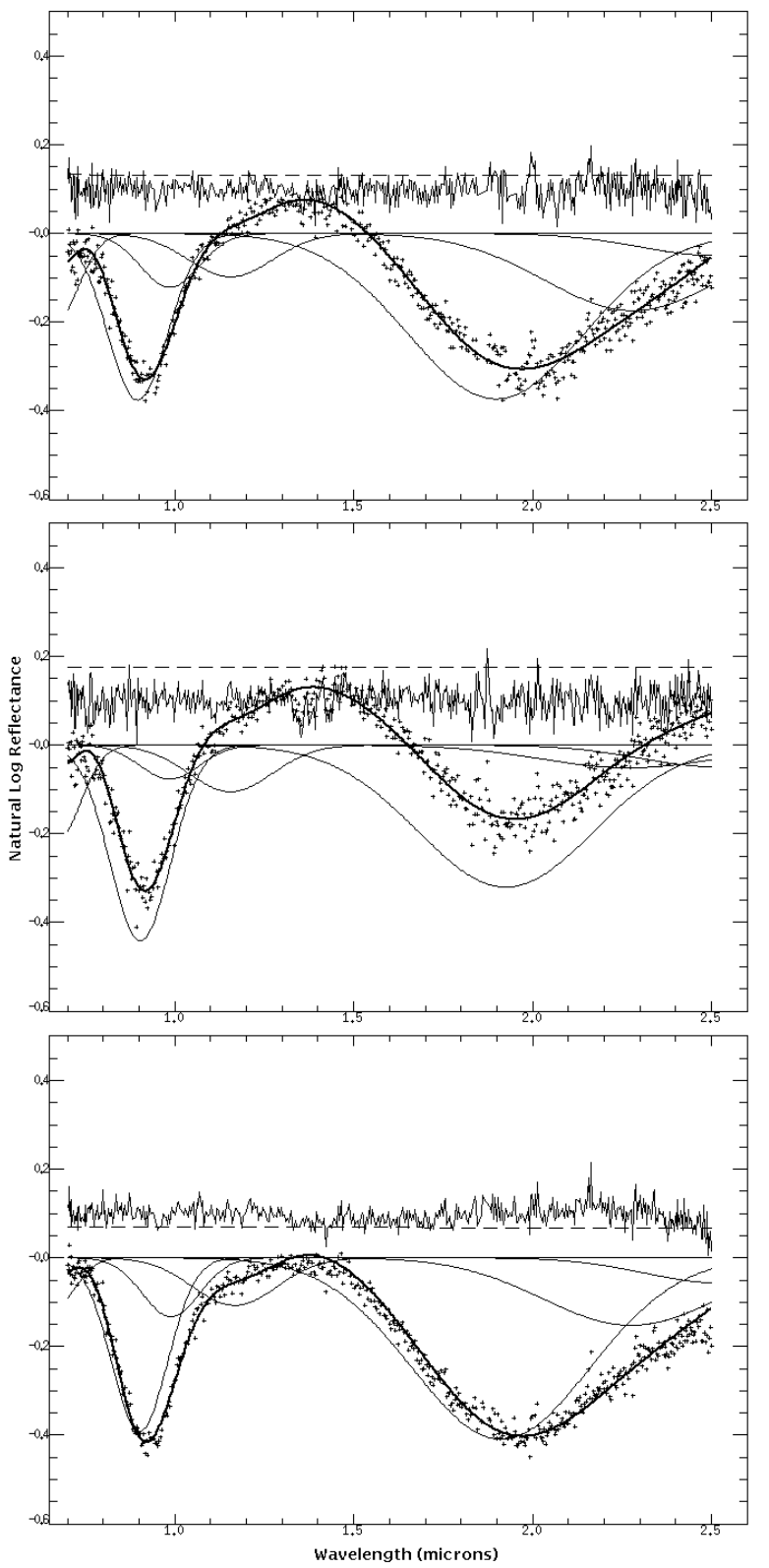

Fig. 4. Spectra of 4 Vesta fitted with seven modified Gaussians (solid lines) corresponding to absorption bands associated to orthopyroxene and clinopyroxene. The dashed line represents the continuum and the residual error spectrum is shown in the upper part of each plot.

of both. The HCP phase could suggest the presence of augite on Vesta, pyroxene that is also found in eucrites. At this point, it is not possible to infer the precise pyroxene compositions using MGM due to the lack of laboratory calibrations for this method.

\subsubsection{Discussion}

The spectral parameters obtained in Sect. 3.1.1 can be directly compared with results of G97 for the asteroid 4 Vesta and also with near-infrared observations both of the V-type asteroids in the vicinity of Vesta (Duffard et al. 2004b) and of 1459 Magnya (Hardersen et al. 2004). 
Table 4. Final parameters from MGM applied to 4 Vesta using both an LCP and an HCP.

\begin{tabular}{|c|c|c|c|c|}
\hline$\Phi=0.06$ & $\begin{array}{c}\text { Center } \\
(\mu \mathrm{m})\end{array}$ & $\begin{array}{c}F W H M \\
(\mu \mathrm{m})\end{array}$ & $\begin{array}{c}\text { Strength } \\
\text { (natural log) }\end{array}$ & $\begin{array}{l}\text { Continuum and } \\
\text { Error in fit (rms) }\end{array}$ \\
\hline Band 1 & 0.658 & 0.145 & -0.11 & \\
\hline Band 2 & 0.902 & 0.183 & -0.39 & Offset:1.1 \\
\hline Band 3 & 0.989 & 0.189 & -0.13 & Slope:1.2E-007 \\
\hline Band 4 & 1.168 & 0.278 & -0.11 & \\
\hline Band 5 & 1.912 & 0.587 & -0.41 & \\
\hline Band 6 & 2.275 & 0.580 & -0.15 & rms: $2.35 \%$ \\
\hline Band 7 & 2.516 & 0.498 & -0.06 & $\%$ \\
\hline \multicolumn{5}{|l|}{$\Phi=0.47$} \\
\hline Band 1 & 0.676 & 0.148 & -0.21 & \\
\hline Band 2 & 0.904 & 0.198 & -0.44 & Offset:1.19 \\
\hline Band 3 & 0.989 & 0.195 & -0.08 & Slope:1.2E-007 \\
\hline Band 4 & 1.157 & 0.276 & -0.10 & \\
\hline Band 5 & 1.925 & 0.579 & -0.32 & rms: $3.3 \%$ \\
\hline Band 6 & 2.270 & 0.580 & -0.05 & \\
\hline Band 7 & 2.513 & 0.500 & -0.05 & $\%$ \\
\hline \multicolumn{5}{|l|}{$\Phi=0.64$} \\
\hline Band 1 & 0.675 & 0.148 & -0.18 & \\
\hline Band 2 & 0.906 & 0.197 & -0.37 & Offset: 1.14 \\
\hline Band 3 & 0.989 & 0.193 & -0.12 & Slope:1.2E-007 \\
\hline Band 4 & 1.158 & 0.277 & -0.10 & \\
\hline Band 5 & 1.922 & 0.583 & -0.39 & rms: $2.93 \%$ \\
\hline Band 6 & 2.306 & 0.580 & -0.15 & \\
\hline Band 7 & 2.515 & 0.499 & -0.05 & $\%$ \\
\hline
\end{tabular}

G97 analysed a set of rotationally-resolved spectrophotometric data of 4 Vesta in the $0.3-2.5 \mu \mathrm{m}$ range obtained in 1981. The author derived a mean pyroxene composition of $\mathrm{Fs}_{46} \mathrm{Wo}_{8}$ (46 mole \% iron and 8\% mole calcium) for Vesta, falling near the low end of the range of values for common eucrites, and considerably above the range of diogenites, with the average surface of Vesta best characterized as a howardite or polymict eucrite. Also, the scatter of the position of BI and BII centers observed by the author was relatively small, with all spectra falling between the eucrite and diogenite loci in the band center diagram. Decreases in BAR values in some rotational phases led the author to suggest the presence of pure olivine regions. In contrast, our data suggest a composition more akin to diogenites with little if any evidence of olivine.

Using the compositional map made from HST observations in the visible range (Binzel et al. 1997), along with the mineralogical map derived by G97, we note that our spectra at rotational phase 0.47 fall on Vesta's eastern hemisphere in a region where the data of both G97 and Binzel et al. (1997) suggest an enhanced diogenitic content. The suggested increase in diogenite contribution at this phase seems to agree with our data, since this rotational phase lies closer to the diogenite locus in the band center diagram, but with the position of BI shifted to lower wavelength than what is seen in G97. The remaining two rotational phases sampled by our spectra lie on
Vesta's western hemisphere, where both G97 and Binzel et al. (1997) suggest the predominance of eucrite-dominated assemblages. This seems consistent with the position of BII for these two phases, but again the BI position for both seem shifted to lower wavelength than G97 spectra at corresponding phases.

Considering that the solar phase angle of two sets of observations are similar and that observations of G97 cover a whole rotational period of Vesta, the differences in the inferred mineralogy must be due to differences in sub-earth latitude of the observations. Using the pole coordinates derived by Thomas et al. (1997), observations of G97 were obtained at a sub-earth latitude of $-20^{\circ}$, while for our observations the sub-earth latitude was $-11^{\circ}$. The slightly more northern sub-earth latitudes of our observations suggest that the region of the crater near the south pole of Vesta might be having less influence on our spectra. This can provide a tentative explanation for the differences between our observations and Gaffey's, because a higher olivine content tends to shift BI to higher wavelength while leaving the position of BII unchanged (Cloutis et al. 1986); and there is both observational evidence (Thomas et al. 1997) and theoretical arguments (Takeda 1979, 1997) for the substantial presence of olivine in the South pole crater of Vesta. A possible problem with this interpretation would be that the BAR values for our spectra are not significantly higher than the ones derived by G97 for most of the surface of Vesta. However, the BAR values can be used to measure olivine content only for olivine/orthopyroxene mixtures, which is not the case for Vesta since the presence of at least two pyroxenes is inferred.

Finally, the present work puts the recent spectral observations of Vestoids in new perspective (Duffard et al. 2004b), as of 1459 Magnya (Hardersen et al. 2004). Duffard et al. (2004b) observed spectra of $19 \mathrm{~V}$-type asteroids in the vicinity of Vesta and found a much greater scatter in BI and BII than what was reported for Vesta by G97. Hardersen et al. (2004) reported that the BI, BII, and BAR values for the asteroid 1459 Magnya were significantly different than G97's results for Vesta, which would rule out the possibility of Magnya being a fragment of Vesta. However, the greater spectral diversity for the surface of Vesta, results from the combination of the spectra presented here with the results of G97, suggests that the spectral differences reported between those asteroids and Vesta do not necessarily imply genetical incompatibility; and this would favour the suggestion of Duffard et al. (2004b) that the spectral diversity observed on the vestoids arise because these asteroids contain material of different layers of Vesta excavated by collisions. As for Magnya, the BI and BII values of our spectra (in particular for phase 0.47) are much closer to Magnya's, which suggests that a genetic connection between Vesta and Magnya cannot be ruled out solely on this basis.

\subsubsection{Mid infrared}

We obtained two spectra of Vesta in the wavelength range 2.0-3.8 $\mu \mathrm{m}$. The smoothed spectra for rotational phases 0.6 and 0.85 are shown in Fig. 5 .

Both spectra present a set of minor absorption features that are more significant at rotational phase 0.6. Those features can 


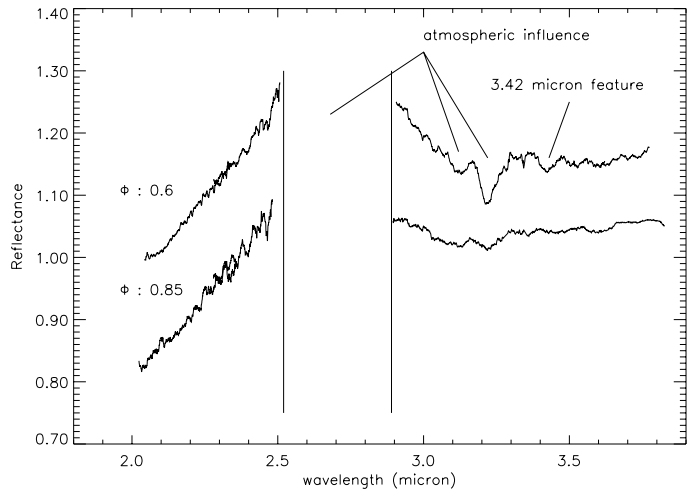

Fig. 5. Spectra of 4 Vesta at two different longitudes after removal of the thermal radiation. Spectra have been brought to lower resolution by Gaussian smoothing. The Gaussian width used was 10 points.

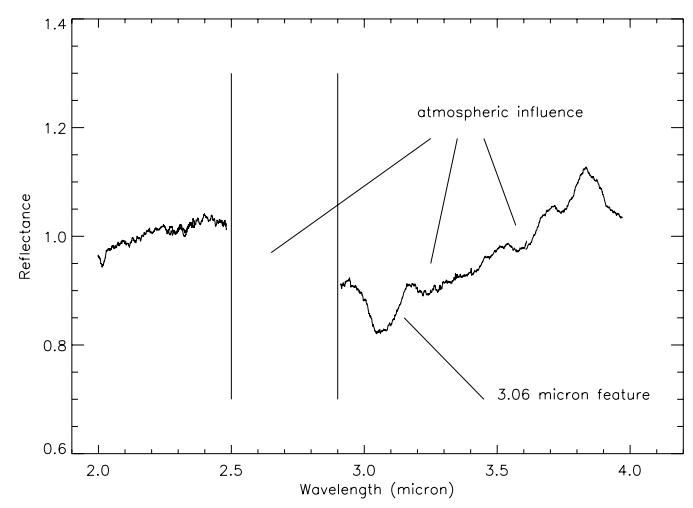

Fig. 6. Spectra of 1 Ceres normalized to one at 2.2 micron and brought to lower resolution by smoothing. The thermal radiation has been removed.

be seen in the atmospheric transmission spectrum and may be due to incomplete correction for atmospheric absorption. The $3.42 \mu \mathrm{m}$ feature seen at rotational phase 0.6 corresponds to a minor atmospheric absorption, and its strength in this spectra is probably due to atmospheric variations during observation.

For any of our spectra, we do not detect an absorption feature at $3.0 \mu \mathrm{m}$, as was seen by Hasegawa et al. (2003) and interpreted as evidence of hydrated and/or hydroxylated minerals on the surface.

We do see a spectral difference between 2.9 and 3.1 micron where the slope of our spectra changes. This variation is possibly due to a difference in pyroxene composition, but we cannot completely exclude that this difference comes from changes in the atmospheric conditions during observations. After $3.1 \mathrm{mi}-$ cron our spectra are almost flat, which is consistent with the spectra of HED meteorites.

\subsection{Ceres}

The spectrum of Ceres obtained in the wavelength range 2.0-4.1 $\mu \mathrm{m}$ is shown in Fig. 6. We calculated the band center and the band depth of the absorption occurring in the $3 \mu \mathrm{m}$ region. The center of the absorption occurs at $3.06 \pm 0.02 \mu \mathrm{m}$, and the depth of the feature is about $10 \%$. These parameters are similar to those found by King et al. (1992).

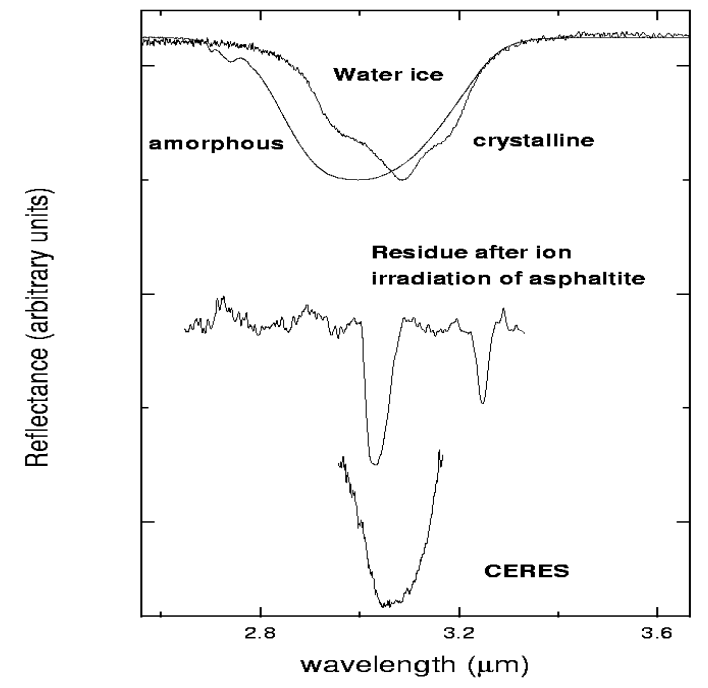

Fig. 7. Spectra of, from the bottom to the top: the $3.06 \mu \mathrm{m}$ band observed on Ceres; an organic refractory residue obtained by ion irradiation of asphaltite covered by water ice $(16 \mathrm{~K})$; amorphous $(16 \mathrm{~K})$; and crystalline $(150 \mathrm{~K})$ water ice. All the spectra are normalized at 1 at their respective maximum and vertically shifted for clarity.

We traced a continuum line between 2.97 and $3.16 \mu \mathrm{m}$, normalized the band at its maximum as shown in Fig. 7 (first spectrum from the bottom), and compared it with a number of different spectra collected in the laboratory at the Osservatorio Astrofisico di Catania (Strazzulla et al. 2001). In particular we compared the observed band with spectra of ices (water, ammonia, methane, methanol, benzene, carbon mono, and di-oxide), ice mixtures, irradiated ices, organic materials (asphaltite, fullerenes, polymers), and residues left over after ion irradiation of carbon bearing species. We obtained the best fit by a linear combination of the spectra of two components: (i) the hemispherical reflectance (room temperature) of an organic refractory residue previously synthesized by ion irradiation of an asphaltite layer covered by water ice (16 K) (see Fig. 7, 2nd spectrum from the bottom); and (ii) the spectrum of crystalline water (at $150 \mathrm{~K}$ ). This is significantly different from the spectrum of amorphous water ice as demonstrated in the same Fig. 7 (top spectrum). Such a difference allows the structure of the ice eventually observed in astronomical objects to be determined.

Asphaltite, a solid bitumen, is very dark in the visual region and has red-sloped spectra in the visible and near-infrared rangen, which may make it a good analog of refractory organic solids on the surfaces of primitive objects from the outer solar system (Moroz et al. 1998). Ion irradiation experiments (Moroz et al. 2004) on bulk samples of pure asphaltite have shown that irradiation-induced carbonization gradually neutralizes spectral slopes of the red organic solids. Asphaltite exhibits several vibrational absorption bands mostly due to aliphatic and aromatic functional groups. Recently experiments of ion irradiation of pure asphaltite and asphaltite covered with water ice were performed (Strazzulla et al. 2004). The results indicate that the original bands of asphaltite progressively decrease in intensity and new bands appear. The spectrum of the asphaltite shown in Fig. 7 refers to a residue obtained after irradiation 


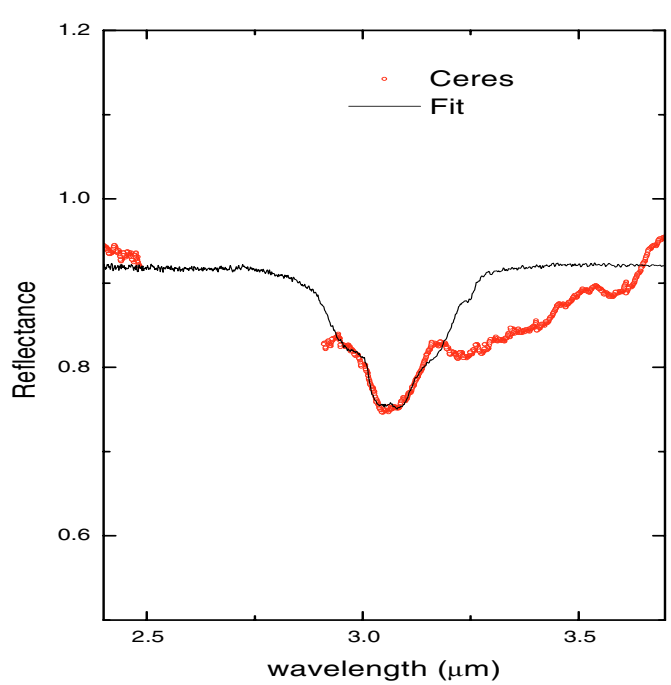

Fig. 8. Comparison of the spectra of the $3.06 \mu \mathrm{m}$ band observed on Ceres and that obtained by a linear combination of the spectra of two laboratory samples as discussed in the text.

(30 keV He$+1.5 \times 10^{16} \mathrm{~cm}^{-2}$ ) of water ice deposited on an asphaltite sample at $16 \mathrm{~K}$. After irradiation the sample has been removed from the vacuum chamber and its spectrum taken at room temperature. Two bands are observed in the 2.7-3.6 $\mu \mathrm{m}$ range. The most intense is centered at $3.03 \mu \mathrm{m}$ and is attributed to $\mathrm{C}-\mathrm{H}$ stretch in carbynoid structures $\left(\mathrm{R}(\mathrm{C} \equiv \mathrm{C})_{\mathrm{m}}-\mathrm{H}\right)$. A second feature, centered at $3.24 \mu \mathrm{m}$, is due to an aromatic $\mathrm{C}-\mathrm{H}$ stretch.

It is well known that ion irradiation produces chemical modifications and, in particular, formation of molecules originally not present in the target. These molecules include species both more volatile and less volatile than the initially irradiated molecular mix. When carbon (or sulfur) is an important constituent of the irradiated material, a refractory residue results and remains after warming the sample. That residue has a complex structure, that, after prolonged irradiation, evolves to form a hydrogenated amorphous carbon. It is interesting to note that the feature at $3.03 \mu \mathrm{m}$ characteristic of linear chains also appears after irradiation of solid acetylene (Strazzulla et al. 2002), solid benzene, and cluster assembled carbon thin films (Strazzulla \& Baratta 1991). Thus its formation seems common to many irradiated hydrogen bearing carbonaceous materials.

In Fig. 8 we compare the spectrum of the band observed on Ceres with that obtained by linear combination of the laboratory spectra discussed above (the two organic residues and crystalline water ice). Features in Ceres' spectrum at $3.20 \mu \mathrm{m}$ and at $3.55 \mu \mathrm{m}$ are due to incomplete removal of telluric features. The fit is very good inside the $3.06 \mu \mathrm{m}$ band, but the mismatch between the fit and spectra after $3.20 \mu \mathrm{m}$ suggests the presence of one or more absorption features in Ceres' spectrum, which are not being reproduced by our model.

We estimated the amount of material that is necessary to give rise to the observed spectrum by measuring the band areas $\left(\mathrm{cm}^{-1}\right)$ on an optical depth scale and dividing their value by the integrated absorbance $\left(\mathrm{cm}\right.$ molecules ${ }^{-1}$; see e.g. Strazzulla et al. (2001) for details). The band areas were measured with respect to a linear continuum that intercepts the spectra at 2.4 and $3.6 \mu \mathrm{m}$. The integrated absorbances we used are $2 \times 10^{-16} \mathrm{~cm} \mathrm{molecules}^{-1}$ for the $\mathrm{O}-\mathrm{H}$ stretches of water ice (Allamandola et al. 1988) and $2 \times 10^{-17} \mathrm{~cm} \mathrm{molecules}^{-1}$ that is both a typical value for the alkyl $\mathrm{CH}_{2}$ and $\mathrm{CH}_{3}$ groups and one we also use for the band at $3.03 \mu \mathrm{m}$. We find that the number of contributing water ice molecules is about $3 \times$ $10^{17}$ molecules $\mathrm{cm}^{-2}$, while the $\mathrm{C}-\mathrm{H}$ groups in linear carbon chains are about $7.5 \times 10^{17} \mathrm{C}-\mathrm{H}$ bonds $\mathrm{cm}^{-2}$.

All of this combines to means that the surface of Ceres (i.e., the outermost skin from which the solar radiation is reflected) contains (i) an organic carbonaceous material with $\mathrm{C}-\mathrm{H}$ bonds in linear chains and alkyl groups; and (ii) a (small) amount of crystalline water ice.

Here we are not claiming that ion irradiation is the only agent that governs the evolution of the Ceres surface. We have used energetic ions in the laboratory to produce processed carbonaceous material that are spectrally very similar to those observed on Ceres. McCord \& Sotin (2003) have suggested that the thermal evolution of Ceres could have produced a metamorphosed carbonaceous chondrite surface material whose ioninduced space weathering would produce the observed features. They also suggested that at least $20 \%$ of Ceres is made of water ice, consistent with the finding of $\mathrm{OH}$ escaping from the north polar region (A'Hearn \& Feldman 1992) and with models indicating that subsurface percolation could replenish the external layers with water ice over geologic time (Fanale \& Salvail 1989). The presence of large amount of water ice has also been suggested recently by Parker et al. (2004) based on measurement (by Hubble Space Telescope) of the equatorial and polar radii of Ceres, whose ratio is consistent with a body that has a central rocky core surrounded by water ice.

\section{Conclusions}

We obtained and analysed spectra for Vesta in the range $0.7-3.8 \mu \mathrm{m}$ and $2.0-4.1 \mu \mathrm{m}$ for Ceres. The near-infrared spectra of Vesta shows a surface composition closer to diogenites than eucrites and possibly a lower olivine content than those obtained by Gaffey (1997). Along with previous works, our results show the surface heterogeneity in greater detail. This seems consistent with recent observations of V-type asteroids in the vicinity of Vesta (Duffard et al. 2004b). We did not find any absorption feature at $3 \mu \mathrm{m}$ indicative of the presence of water ice. For Ceres we confirm the presence of the absorption feature at $3.06 \mu \mathrm{m}$ that was previously reported (Lebofsky et al. 1981; King et al. 1992) and we suggest that this feature can be reproduced with a linear combination of crystaline water ice and residues of ion-irradiated asphaltite covered with water ice. The presence of organic materials, in particular of carbon linear chain present in asphaltite, was never reported earlier in any asteroid. If real, it would confirm the uniqueness of the Ceres surface.

This work was support for the Dawn mission that will provide detailed mineralogical maps for Vesta and Ceres. However, in order to make a good assessment of the data, more laboratory calibrations are needed, in particular precise deconvolution of the HED meteorites using MGM.

Acknowledgements. T. Mothé-Diniz wishes to thank CNPq (Conselho Nacional de Desenvolvimento Científico e Tecnológico) for the 
fellowship PDE that contributed to realization of this work. We thank the referee D. Cruikshank for his pertinent and constructive remarks.

\section{References}

A'Hearn, M. F., \& Feldman, P. D. 1992, Icarus, 98, 54

Allamandola, L. J., Sandford, S. A., \& Valero, G. J. 1988, Icarus, 76, 225

Binzel, R. P., Gaffey, M. J., Thomas, P. C., et al. 1997, Icarus, 128, 95

Birlan, M., Binzel, R., Bus, S., et al. 2002, BAAS, 34, 860

Britt, D. T., Yeomans, D., Housen, K., \& Consolmagno, G. 2002, in Asteroids III (University of Arizona Press), 485

Cloutis, E. A., \& Gaffey, M. J. 1991, J. Geophys. Res., 96, 22809

Cloutis, E. A., Gaffey, M. J., Jackowski, T. L., \& Reed, K. L. 1986, J. Geophys. Res., 91, 11641

Duffard, R., Lazzaro, D., \& De Leon, J. 2004a, Meteoritics and Planetary Science

Duffard, R., Lazzaro, D., Licandro, J., et al. 2004b, Icarus, 171, 120

Fanale, F. P., \& Salvail, J. R. 1989, Icarus, 82, 97

Gaffey, M. J. 1997, Icarus, 127, 130

Gaffey, M. J., Cloutis, E. A., Kelley, M. S., \& Reed, K. L. 2002, in Asteroids III (University of Arizona Press), 183

Hardersen, P. S., Gaffey, M. J., \& Abell, P. A. 2004, Icarus, 167, 170

Hasegawa, S., Murakawa, K., Ishiguro, M., et al. 2003, Geophys. Res. Lett., 30, 2

Keil, K. 2002, in Asteroids III (University of Arizona Press), 573

King, T. V. V., Clark, R. N., Calvin, W. M., Sherman, D. M., \& Brown, R. H. 1992, Science, 255, 1551

Lebofsky, L. A., Feierberg, M. A., Tokunaga, A. T., Larson, H. P., \& Johnson, J. R. 1981, Icarus, 48, 453

Lebofsky, L. A., \& Spencer, J. R. 1989, in Asteroids II (University of Arizona Press), 128
McCord, T. B., Adams, J. B., \& Johnson, T. V. 1970, Science, 168, 1445

McCord, T. B., \& Sotin, C. 2003, AAS/Division for Planetary Sciences Meeting, 35

Moroz, L., Baratta, G., Strazzulla, G., et al. 2004, Icarus, 170, 214

Moroz, L. V., Arnold, G., Korochantsev, A. V., \& Wasch, R. 1998, Icarus, 134, 253

Parker, J. W., Stern, S. A., Thomas, P. C., et al. 2002, AJ, 123, 549

Parker, J. W., Thomas, P., Young, E., et al. 2004, AAS/Division for Planetary Sciences Meeting, 36,

Standish, E. M., \& Hellings, R. W. 1989, Icarus, 80, 326

Strazzulla, G., \& Baratta, G. A. 1991, A\&A, 241, 310

Strazzulla, G., Baratta, G. A., \& Palumbo, M. E. 2001, Spectrochim. Acta, 57, 825

Strazzulla, G., Baratta, G. A., Domingo, M., \& Satorre, M. A. 2002, Nucl. Instr. Methods Phys. Res. B, 191, 714

Strazzulla, G., Battiato, S., \& Compagnini, G. 2004 (New York), in press

Sunshine, J. M., \& Pieters, C. M. 1993, J. Geophys. Res., 98, 9075

Sunshine, J. M., Pieters, C. M., \& Pratt, S. F. 1990, J. Geophys. Res., 95, 6955

Sunshine, J. M., Bus, S. J., McCoy, T. J., et al. 2004, Meteoritics and Planetary Sci., 39, 1343

Takeda, H. 1979, Icarus, 40, 455

Takeda, H. 1997, Meteoritics and Planetary Sci., 32, 841

Tedesco, E. F. 1989, in Asteroids II (University of Arizona Press), 1090

Thomas, P. C., Binzel, R. P., Gaffey, M. J., et al. 1997, Science, 277, 1492

Webster, W. J., Johnston, K. J., Hobbs, R. W., et al. 1988, AJ, 95, 1263 